\title{
Hemophagocytic Lymphohistiocytosis as a Manifestation of Underlying Visceral Leishmaniasis
}

Michael D. Diamantidis ${ }^{1}$, Andromachi Palioura ${ }^{2}$, Maria Ioannou ${ }^{3}$, Evangelos Tsangalas ${ }^{1}$, Konstantinos Karakousis Sr. ${ }^{2}$

1. Hematology, First Department of Internal Medicine, Thalassemia and Sickle Cell Disease Unit, General Hospital of Larissa, Larissa, GRC 2. Internal Medicine, First Department of Internal Medicine, General Hospital of Larissa, Larissa, GRC 3. Pathology, Haemopathology, University Hospital of Larissa, University of Thessaly, Larissa, GRC

Corresponding author: Michael D. Diamantidis, diamantidis76@gmail.com

\begin{abstract}
Hemophagocytic lymphohistiocytosis (HLH), or hemophagocytic syndrome (HS) is a severe syndrome involving an extreme participation of the immune system, resulting in a cascade of cytokines, hyperinflammation and extensive hemophagocytosis in the bone marrow (BM) and affecting the peripheral blood (PB) lineages. Fever, splenomegaly, hypertriglyceridemia, hypofibrinogenemia, and hyperferritinemia are often encountered in this disease. The syndrome can be seen in all ages and it is either primary due to genetic defects or secondary because of malignancies, immune deficiencies, rheumatic diseases, and infections. Bacteria, viruses, protozoa, and fungi are often implicated. Visceral leishmaniasis (VL) is among the infectious causes of HLH. We describe a patient with a successful treatment of HLH after the initiation of liposomal amphotericin B, due to $\mathrm{VL}$, even though there was a delay in diagnosing the leishmaniasis. The exact precipitating pathophysiological events triggering HLH remain unknown and provide their clear impact for future research. An instructive, critical review of the literature related to the presented case is provided. Distinguishing secondary HS from primary HS is essential for the application of suitable treatment. Improper use of corticosteroids could cover up an underlying possible malignancy or infection and delay the initiation of the etiologic therapeutic strategy.
\end{abstract}

Review began $11 / 28 / 2020$ Review ended 12/01/2020 Published 12/04/2020

\section{(๑) Copyright 2020}

Diamantidis et al. This is an open access article distributed under the terms of the Creative Commons Attribution License CC-BY 4.0., which permits unrestricted use, distribution, and reproduction in any medium, provided the original author and source are credited.
Categories: Internal Medicine, Infectious Disease, Hematology

Keywords: hemophagocytic lymphohistiocytosis (hlh), hemophagocytic syndrome (hs), macrophage activation syndrome (mas), visceral leishmaniasis (vl), liposomal amphotericin b

\section{Introduction}

The etymology of the word "hemophagocytosis" comes from the combination of the Greek words haema (=blood), phago (=eat), and cytos (=container). It is used to describe the pathological condition of several types of cells in the bone marrow (BM), usually macrophages, destroying other cells or large particles by engulfing them with their plasma membrane, giving rise to an internal compartment, called the phagosome.

Hemophagocytic lymphohistiocytosis (HLH) is an aggressive and life-threatening syndrome of excessive immune activation observed in children and adults of all ages. It is also called hemophagocytic syndrome (HS) or macrophage activation syndrome (MAS). More precisely, the clinical syndrome caused by HLH should be named HS. Nevertheless, there is often overlap in the literature between the two terms, both referring to the same life-threatening condition.

HLH can occur as a familial or sporadic disorder, and might be triggered by a variety of events that disrupt immune homeostasis. Infection is a common trigger both in those with a genetic predisposition and in sporadic cases. Genetic or primary HLH has been linked to distinct molecular or chromosomal aberrations [1]. Acquired or secondary HLH derives either from exogenous agents, such as infectious organisms or toxins, or from endogenous products, related to tissue damage, radical stress, and metabolic products [2]. Rheumatic diseases, immune deficiencies [1], and malignancies, such as lymphomas or multiple myeloma, can also trigger HLH.

Interestingly, HLH secondary to disseminated histoplasmosis in systemic lupus erythematosus [3] or in patients with human immunodeficiency virus (HIV) $[4,5]$ has been described. Moreover, from the newest immunomodulatory monoclonal antibodies, the drug daratumumab has been implicated for inducing HLH as well [6].

Visceral leishmaniasis (VL) is among the infectious causes of HLH. The diagnosis can sometimes be tricky. We describe a case of HLH as a manifestation of VL.

\section{Case Presentation}


An 82-year-old female with arterial hypertension controlled with ramipril presented to our center with fever $\left(38.6^{\circ} \mathrm{C}\right)$ for four days without chills. She reported mild weakness with unclear onset and lacked other Bsymptoms, such as night sweats or weight loss. Laboratory results showed pancytopenia (white blood cells (WBCs) $1,600 / \mu \mathrm{L}$, neutrophils $970 / \mu \mathrm{L}$, lymphocytes $580 / \mu \mathrm{L}$, monocytes $40 / \mu \mathrm{L}$, hemoglobin $10.7 \mathrm{~g} / \mathrm{dL}$, platelets $85,000 / \mu \mathrm{L}$ ). She was admitted to the Internal Medicine Department for further evaluation and treatment.

Additional diagnostic tests showed increased ferritin $(7,146 \mathrm{ng} / \mathrm{ml}$ [normal $<307 \mathrm{ng} / \mathrm{ml}])$, triglycerides [390 $\mathrm{mg} / \mathrm{dl}$ (normal <150 mg/dl)] and lactate dehydrogenase (LDH) levels [1,358 U/L (normal <480 U/L)].

Fibrinogen levels were low [134 mg/dl (normal 200-400)]. After two days, fibrinogen further decreased (64 $\mathrm{mg} / \mathrm{dl})$, while ferritin improved slightly $(5,167 \mathrm{ng} / \mathrm{ml})$, still much above the normal range. Empirical treatment with ampicillin plus tazobactam was initiated, whereas three consequent blood cultures under fever from peripheral blood were negative. A complete infection work-up (Epstein-Barr virus (EBV), cytomegalovirus (CMV), herpes simplex virus (HSV), hepatitis B virus (HBV), hepatitis C virus (HCV), HIV, toxoplasma, Widal, Wright) was also negative. The patient had no splenomegaly. Chest and abdomen CT showed no significant radiological findings or lymphadenopathy. Because of the laboratory data, hemophagocytic syndrome was suspected.

On day five, bone marrow (BM) aspiration yielded extensive hemophagocytosis by histiocytes of erythroblasts, red cells, platelets, and remnants of nuclei resembling monocytes (Figure 1, A-C). Precursors of all cell lines were also eliminated by the phagocytes (Figure 1, A-C). Parasites resembling leishmanias were observed and an additional screening for leishmaniasis, which involved polymerase chain reaction (PCR), antibodies for leishmanias plus a pending BM biopsy, was recommended.
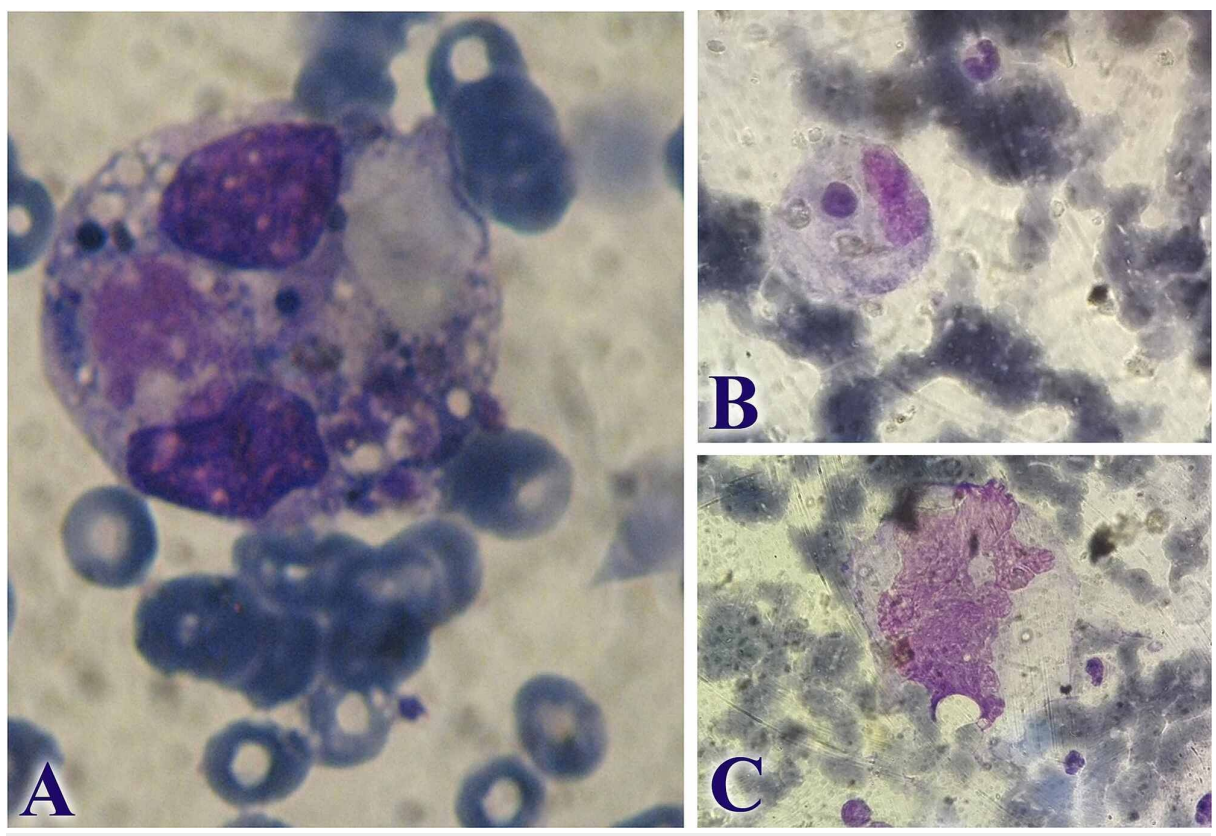

\section{FIGURE 1: Bone Marrow Aspiration}

A. Extensive hemophagocytosis by histiocytes of erythroblasts, red cells, platelets and remnants of nuclei resembling monocytes; B. Hemophagocytosis of cellular elements at a lesser degree; C. Hemophagocytosis of a cellular element resembling a remnant of a megakaryocyte.

Thus, five out of eight criteria for HLH (fever, cytopenias involving two lineages, hypertriglyceridemia and hypofibrinogenemia, hemophagocytosis in BM and hyperferritinemia) were fulfilled [7]. Measuring a possible low natural killer (NK) cell activity and the soluble CD25 [interleukin (IL)-2 receptor] levels were not available in our department and were not conducted. Even though the leishmania parasites were observed in the BM aspirate, etiological treatment for the infection was not initiated, due to the pending BM biopsy. On day six, the suppression of the excessive activation of the immune system was proposed as a therapeutic strategy and intravenous prednisolone at the dosage of $25 \mathrm{mg}$, three times daily, was initiated. As the fever persisted, ampicillin and tazobactam were discontinued and ceftriaxone plus meropenem were applied.

On day 10, antibodies for leishmanias with the technique of immunochromatography were positive and a history of direct contact of the patient with canines was discovered. On day 13, platelets further decreased to $55,000 / \mu \mathrm{L}$, while on day 16 , ferritin levels reached their highest value of $10,500 \mathrm{ng} / \mathrm{ml}$ and $\mathrm{LDH}$ was 1,873 $\mathrm{U} / \mathrm{L}$. Human normal immunoglobulins $[300 \mathrm{mg} / \mathrm{kg}(5 \mathrm{~g} / 100 \mathrm{ml})]$ were applied as an attempt to control the 
thrombocytopenia, but with no result. A polyclonal increase of immunoglobulin (Ig) A and IgM immunoglobulins was observed, as expected in leishmaniasis. On day 17 , platelets were as low as 30,000/ $\mu \mathrm{L}$.

Finally, 19 days after the initial admission of the patient, BM trephine biopsy showed cells with granules, suspect for intracellular parasite of the type of leishmania (Figure 2, A-B). A positive PCR for leishmanias in the BM further confirmed the diagnosis of VL. Even though there was strong suspicion for leishmaniasis from day five (BM aspiration), the final diagnosis was established on day 19 and etiological treatment was initiated on day 23.
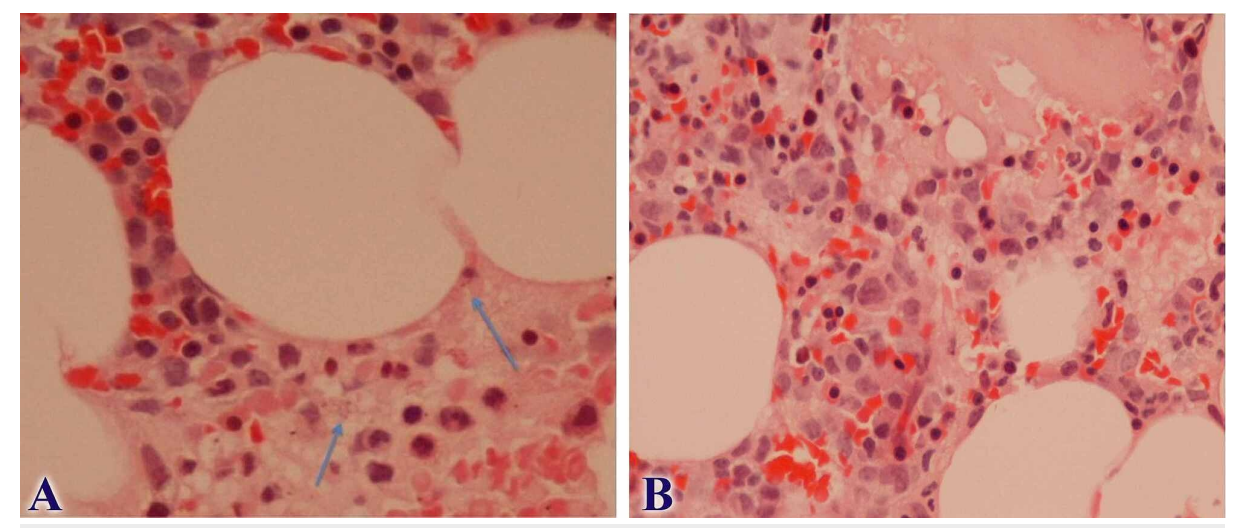

FIGURE 2: Bone Marrow Biopsy

A. Cells with granules, suspect for intracellular parasite of the type of leishmania (blue arrows). Hematoxylin and Eosin (HE) stain X 400; B. Overall imaging of the bone marrow showing leishmanias. Hematoxylin and Eosin (HE) stain X 100

Hence, liposomal amphotericin B (light-sensitive drug) was applied at a daily dosage of $3 \mathrm{mg} / \mathrm{kg}$ for five days. An improvement in the laboratory data was observed from day 24 and the fever subsided on day 26 (platelets $72,000 / \mu \mathrm{L}$, triglycerides $316 \mathrm{mg} / \mathrm{dl}$, ferritin $3,948 \mathrm{ng} / \mathrm{ml}$ ). On day 27 , she was discharged from the hospital with oral methylprednisolone ( $32 \mathrm{mg}$ daily). A complete recovery of the clinical manifestations and a vast improvement of the laboratory findings were finally seen on day 34 (ferritin $846 \mathrm{ng} / \mathrm{ml}$, fibrinogen 182 $\mathrm{mg} / \mathrm{dl}$ ). Daily sessions of liposomal amphotericin B every 15 days for the next three months (maintenance therapy, six cycles totally) were applied. The patient remains afebrile and healthy with complete resolution of VL until today, at the age of 88 .

\section{Discussion}

Several molecular mechanisms for HLH have been proposed. The clinical presentation of the patient is not directly ascribed to VL pathogenesis and only molecular postulations can be made linking VL and HLH. An abnormal activation of monocytes, macrophages, dendritic cells, cytotoxic NK- or T-cells has been implicated [2]. An uncontrolled and ineffective immune response is the hallmark of HS [8]. More specifically, impaired perforin-dependent cytotoxicity, impaired control of infections, as well as dysregulated inflammasome activity affecting IL-1 $\beta$ and IL-18, are all examples of the cascade of abnormal function of the immune system in this syndrome [9]. Next generation sequencing has identified familial HLH genes, variants in primary immunodeficiency (PIDD)-associated genes or dysregulated immune activation or proliferation (DIAP) genes [10].

Zoonotic diseases are an important cause of HLH. Brucellosis, rickettsial diseases and Q fever are the most common bacterial etiologies. Regarding viral diseases, patients with avian influenza A subtype H5N1 and EBV infections are frequently implicated in the pathogenesis of HLH. Among the protozoa, most of the cases were reported in patients with VL. Regarding fungi, most of the cases were reported in HIV patients with histoplasmosis [11].

In the pandemic coronavirus disease 2019 (COVID-19) infection, the increased levels of inflammatory cytokines (hyperinflammation, cytokine storm syndrome) resemble the cytokine profile observed in cases of secondary HLH and MAS [12]. Severe hemophagocytosis on BM aspirates, cytopenia of two or more lineages and increased serum ferritin levels ( $\geqslant 2000 \mathrm{ng} / \mathrm{mL}$ ) were shown in patients with severe COVID-19 [13]. The control of hypercytokinemia is the key to successfully treat secondary HLH/MAS. However, the effectiveness of cytokine blocking with anti-IL-1 and anti-IL-6 on secondary HLH/MAS has been limited [12,13].

VL is a rare cause of HS, linked to HLH. Several cases have been reported in the literature [14,15]. An unusual manifestation of VL mimicking lymphoma has also been described [16]. In most of these cases the diagnosis was delayed because the clinical suspicion for leishmaniasis as a trigger for HLH involves an 
experienced hematologist or hematopathologist in the BM morphology. The infusion of immunoglobulins did not improve the patient's thrombocytopenia, because the latter was due to the infection. The delay for initiation of the etiological treatment was because of the pending BM biopsy, for the confirmation of the presence of the parasites in the BM, besides the aspiration. Various diagnostic assays for visceral leishmaniasis with diverse degrees of specificity and sensitivity have been proposed [16]; however, the gold standard remains the visual identification of the parasite in the BM. Nevertheless, due to the life cycle of the parasite, the presence of protozoa can be inconsistent between consecutive BM instances/images. The success rate of the initiation of amphotericin B is very high for treating VL [14].

In general, HS deriving from secondary HLH has a grave prognosis [17]. However, in recent years, the outcome of HLH has improved, due to a better molecular understanding of the syndrome, along with the initiation of intensive chemo- and immunotherapy. The prognosis of HLH in children differs by HLH subtype. In Japan, EBV-HLH is the most common HLH subtype [18]. Patients with relapsed or refractory disease after the application of the HLH-2004 protocol and those with the familial subtype should be treated with hematopoietic stem cell transplantation (HSCT) [17,18].

In cases of superficial interpretation of the diagnostic criteria for HLH, a secondary HLH due to malignancy or infection might incorrectly be considered as a primary HLH. Improper use of corticosteroids could cover up an underlying possible malignancy or infection and delay the initiation of the suitable treatment. A coexistence of both cancer and infection behind a secondary HLH should be excluded (EBV driver of lymphoma).

The specificity of the criteria for differential diagnosis between primary and secondary HLH is based on 2004 laboratory techniques and has not been extensively studied. These criteria are helpful in diagnosing the primary cause of HLH only in $20 \%$ of cases $[19,20]$. The optimal treatment of HLH remains unknown to this day. The inhibition of hyper-inflammation with immunosuppression is necessary; however this strategy might decrease the defense mechanisms against a possible primary infectious factor. Prolonged immunosuppression could lead to a reactivation of the initial trigger of $\mathrm{HLH}$, thereby leading to a vicious cycle $[8,19]$. Clinicians should be cautious in distinguishing secondary HS from primary HS and should also define the underlying causes behind secondary HLH. VL must always be considered among the infectious etiologies resulting in HLH.

\section{Conclusions}

We describe a case of HLH as a manifestation of VL. The clinical suspicion for leishmaniasis involves an experienced hematologist in the BM morphology. Due to the life cycle of the parasite, the presence of protozoa can be inconsistent between subsequent BM instances. In cases of superficial interpretation of the diagnostic criteria for HLH, a secondary HLH due to malignancy or infection might incorrectly be considered as a primary HLH. Thus, clinicians should be cautious in distinguishing secondary HS from primary HS.

\section{Additional Information \\ Disclosures}

Human subjects: Consent was obtained by all participants in this study. Conflicts of interest: In compliance with the ICMJE uniform disclosure form, all authors declare the following: Payment/services info: All authors have declared that no financial support was received from any organization for the submitted work. Financial relationships: All authors have declared that they have no financial relationships at present or within the previous three years with any organizations that might have an interest in the submitted work. Other relationships: All authors have declared that there are no other relationships or activities that could appear to have influenced the submitted work.

\section{Acknowledgements}

The authors would like to thank Dr. Theodora Konstantoula and Dr. Konstantinos Krapis for treating the patient and Prof. Dr. Efthymia Petinaki for the PCR analysis of the leishmanias in the BM. The authors would also like to thank the Microbiologists, Directors of the Department of Microbiology of the General Hospital of Larissa, Drs. Lampiri Vassiliki, Papachatzi Maria and Zisis Nikolaos for the laboratory workout of the patient. M.D.D. diagnosed VL in the BM aspirate, analyzed and interpreted data, provided the images of the BM aspirate, performed literature review, wrote and corrected the manuscript; A.P., and K.Karakousis treated the patient, analyzed and interpreted data; M.I. analyzed and interpreted data, provided the pathology images of the BM biopsy and diagnosed the leishmaniasis in the BM biopsy; E.T. wrote the manuscript, analyzed and interpreted data, performed literature review and edited the references

\section{References}

1. Janka GE: Hemophagocytic syndromes. Blood Rev. 2007, 21:245-253. 10.1016/j.blre.2007.05.001

2. Brisse E, Wouters $\mathrm{CH}$, Matthys $\mathrm{P}$ : Advances in the pathogenesis of primary and secondary haemophagocytic lymphohistiocytosis: differences and similarities. Br J Haematol. 2016, 174:203-217. 10.1111/bjh.14147

3. Souto Filho JD, Lima PA, Paulo AB, Souza AS: Hemophagocytic lymphohistiocytosis secondary to 
disseminated histoplasmosis in systemic lupus erythematosus. Int J Hematol. 2017, 106:727-728. 10.1007/s12185-017-2308-z

4. Gil-Brusola A, Pemán J, Santos M, Salavert M, Lacruz J, Gobernado M: Disseminated histoplasmosis with hemophagocytic syndrome in a patient with AIDS: description of one case and review of the Spanish literature. Rev Iberoam Micol. 2007, 24:312-316. 10.1016/s1130-1406(07)70063-3

5. De Lavaissière $M$, Manceron $\mathrm{V}$, Bourée $\mathrm{P}$, et al.: Reconstitution inflammatory syndrome related to histoplasmosis, with a hemophagocytic syndrome in HIV infection. J Infect. 2009, 58:245-247. 10.1016/j.jinf.2008.11.010

6. Woods A, Wooten M, Thompson Heffner L, Waller E: Daratumumab-associated hemophagocytic lymphohistiocytosis. Ann Hematol. 2020, 99:181-182. 10.1007/s00277-019-03877-7

7. Henter JI, Horne A, Aricó M, et al.: HLH- 2004: Diagnostic and therapeutic guidelines for hemophagocytic lymphohistiocytosis. Pediatr Blood Cancer. 2007, 48:124-131. 10.1002/pbc.21039

8. Janka GE, Lehmberg K: Hemophagocytic lymphohistiocytosis: pathogenesis and treatment. Hematology Am Soc Hematol Educ Program. 2013, 2013:605-611. 10.1182/asheducation-2013.1.605

9. Sepulveda FE, de Saint Basile G: Hemophagocytic syndrome: primary forms and predisposing conditions . Curr Opin Immunol. 2017, 49:20-26. 10.1016/j.coi.2017.08.004

10. Chinn IK, Eckstein OS, Peckham-Gregory EC, et al.: Genetic and mechanistic diversity in pediatric hemophagocytic lymphohistiocytosis. Blood. 2018, 132:89-100. 10.1182/blood-2017-11-814244

11. Cascio A, Pernice LM, Barberi G, et al.: Secondary hemophagocytic lymphohistiocytosis in zoonoses. A systematic review. Eur Rev Med Pharmacol Sci. 2012, 16:1324-1337.

12. Takami A: Possible role of low-dose etoposide therapy for hemophagocytic lymphohistiocytosis by COVID19. Int J Hematol. 2020, 112:122-124. 10.1007/s12185-020-02888-9

13. Mehta P, McAuley DF, Brown M, Sanchez E, Tattersall RS, Manson JJ: COVID- 19: consider cytokine storm syndromes and immunosuppression. Lancet. 2020, 395:1033-1034. 10.1016/s0140-6736(20)30628-0

14. Rajagopala S, Dutta U, Chandra KS, Bhatia P, Varma N, Kochhar R: Visceral leishmaniasis associated hemophagocytic lymphohistiocytosis--case report and systematic review. J Infect. 2008, 56:381-388. 10.1016/j.jinf.2008.02.013

15. Koubâa M, Mâaloul I, Marrakchi C, et al.: Hemophagocytic syndrome associated with visceral leishmaniasis in an immunocompetent adult-case report and review of the literature. Ann Hematol. 2012, 91:1143-1145. 10.1007/s00277-011-1367-0

16. Evers G, Pohlen M, Berdel WE, et al.: Visceral leishmaniasis clinically mimicking lymphoma. Ann Hematol. 2014, 93:885-887. 10.1007/s00277-013-1896-9

17. Park HS, Kim DY, Lee JH, et al.: Clinical features of adult patients with secondary hemophagocytic lymphohistiocytosis from causes other than lymphoma: an analysis of treatment outcome and prognostic factors. Ann Hematol. 2012, 91:897-904. 10.1007/s00277-011-1380-3

18. Yanagisawa R, Nakazawa Y: Outcomes in children with hemophagocytic lymphohistiocytosis treated using HLH-2004 protocol in Japan. Int J Hematol. 2019, 109:206-213. 10.1007/s12185-018-02572-z

19. Gurunathan A, Boucher AA: Limitations of HLH-2004 criteria in distinguishing malignancy-associated hemophagocytic lymphohistiocytosis. Pediatr Blood Cancer. 2018, 65:27400.

20. Brisse E, Matthys P, Wouters CH: Understanding the spectrum of haemophagocytic lymphohistiocytosis: update on diagnostic challenges and therapeutic options. Br J Haematol. 2016, 174:175-187. 10.1111/bjh.14144 